\title{
O uso do acido hialurônico na artroscopia: revisão sistemática
}

\author{
The use of hyaluronic acid in arthroscopy: systematic review \\ El uso de ácido hialurónico en artroscopia: revisión sistemática
}

Recebido: 16/08/2021 | Revisado: 29/09/2021 | Aceito: 10/09/2021 | Publicado: 12/09/2021

\author{
Igor Leitão Marques \\ ORCID: https://orcid.org/0000-0002-9921-0627 \\ São Leopoldo Mandic, Brasil \\ E-mail: drigorlmarques@gmail.com.br \\ Marília de Oliveira Coelho Dutra Leal \\ ORCID: https://orcid.org/0000-0003-1164-9983 \\ São Leopoldo Mandic, Brasil \\ E-mail: marilialeal@hotmail.com \\ Cláudio Roberto Pacheco Jodas \\ ORCID: https://orcid.org/0000.0002-9750-2714 \\ São Leopoldo Mandic, Brasil \\ E-mail: claudio.jodas@ slmandic.edu.br \\ Rubens Gonçalves Teixeira \\ ORCID: https://orcid.org/0000-0003-0814-0940 \\ São Leopoldo Mandic, Brasil \\ E-mail: rgte@terra.com.br
}

\begin{abstract}
Resumo
O objetivo do presente estudo foi avaliar a efetividade do uso de injeções de ácido hialurônico em procedimentos de artroscopia em pacientes com desordens temporomandibulares (DTMs). A metodologia empregada para alcançar o intuito proposto foi uma revisão da literatura atualmente disponível e conduzida nas bases de dados MEDLINE via PubMed, Scopus, Scielo e Bireme, por meio de termos controlados e não controlados, com o uso de operadores booleanos e Descritores em Ciências da Saúde DeCS/MeSH previamente escolhidos. Após a aplicação das estratégias de busca e critérios de elegibilidade, 13 artigos foram incluídos e as características foram coletadas: autor, ano, tipo de estudo, metodologia de diagnóstico de DTM, tamanho amostral, ácido hialurônico utilizado, uso de outros agentes terapêuticos, tempo de acompanhamento e benefícios relatados. A interpretação das evidências obtidas nesse estudo permitiu concluir que o uso de ácido hialurônico em procedimentos de artroscopia é efetiva e pode ser recomendada para pacientes em tratamento para DTMs. Foi evidenciada a necessidade de mais estudos acerca do tema, a fim de estabelecer um protocolo padrão para os atendimentos.
\end{abstract}

Palavras-chave: Articulação temporomandibular; Desordem temporomandibular; Artroscopia; Ácido hialurônico; Viscossuplementação.

\begin{abstract}
The aim of the present study was to evaluate the effectiveness of using hyaluronic acid injections after arthroscopic procedures in patients with temporomandibular disorders. The methodology used to achieve the proposed aim was a literature review currently available and conducted in MEDLINE databases via PubMed, Scopus, Scielo and Bireme, through controlled and uncontrolled terms, using Boolean operators and Descriptors in Sciences of the Health DeCS/MeSH previously chosen. After applying the search strategies and eligibility criteria, 13 articles were included and the following data were collected: author, year, study type, methodology for diagnosing temporomandibular disorder, sample size, hyaluronic acid used, use of other therapeutic agents, follow-up period and reported benefits. Interpretation of the evidence obtained in this study allowed us to conclude that the use of hyaluronic acid in arthroscopy procedures is effective and can be recommended for patients being treated for temporomandibular disorders. The need for further studies on the subject was highlighted, to establish a care standard protocol.
\end{abstract}

Keywords: Temporomandibular joint; Temporomandibular disorder; Arthroscopy; Hyaluronic acid; Viscosupplementation.

\section{Resumen}

El objetivo del presente estudio fue evaluar la efectividad del uso de inyecciones de ácido hialurónico en procedimientos artroscópicos en pacientes con trastornos temporomandibulares (TTM). La metodología utilizada para lograr el objetivo propuesto fue una revisión de la literatura actualmente disponible y realizada en las bases de datos MEDLINE vía PubMed, Scopus, Scielo y Bireme, mediante términos controlados y no controlados, utilizando operadores booleanos y Science Descriptors of Health DeCS / MeSH previamente elegidos. Luego de aplicar las estrategias de búsqueda y los criterios de elegibilidad, se incluyeron 13 artículos y se recolectaron las características: autor, año, tipo de estudio, metodología de diagnóstico de TTM, tamaño de muestra, ácido hialurónico utilizado, uso 
de otros agentes terapéuticos, seguimiento del tiempo y beneficios reportados. La interpretación de la evidencia obtenida en este estudio nos permitió concluir que el uso de ácido hialurónico en procedimientos artroscópicos es efectivo y puede ser recomendado para pacientes en tratamiento de TMD. Se destacó la necesidad de realizar más estudios sobre el tema, con el fin de establecer un protocolo estándar de atención.

Palabras clave: Articulación temporomandibular; Trastorno temporomandibular; Artroscopia; Ácido hialurónico; Viscosuplementación.

\section{Introdução}

As desordens temporomandibulares (DTMs) são um conjunto de disfunções e transtornos que acometem integrantes do sistema estomatognático, principalmente os músculos da mastigação, ATM (Articulação Temporomandibular) e suas estruturas associadas (Grossmann, Januzzi, \& Iwaki Filho, 2013; Sousa, López-Valverde, López-Valverde, Caramelo, Fraile, Payo, 2020). A DTM apresenta uma alta incidência e prevalência, sendo mais encontrada em indivíduos adultos jovens, do sexo feminino (Cömert Kiliç, Güngörmüş, 2015).

A DTM pode ter acometimento muscular e/ou articular. Dentre as desordens do complexo disco articular, é possível subdividi-las em deslocamentos de disco com redução e deslocamentos de disco sem redução (Grossmann et al., 2013). Há ainda as desordens degenerativas da ATM como osteoartrites e osteoartroses (Castaño-Joaqui, Cano-Sánchez, Campo-Trapero, \& Muñoz-Guerra, 2021; Grossmann et al., 2013) e inflamatórias como sinovites, retrodiscites e capsulites (Bonotto, Custódio, \& Cunali, 2011).

A osteoartrite pode ser conceituada como uma doença de caráter crônico e não inflamatório, caracterizada pela degradação progressiva do disco articular, remodelação óssea subcondral e inflamação crônica no tecido sinovial das articulações (Liu et al., 2018).

Prioritariamente, o protocolo padrão de tratamento para DTM inicia-se de forma conservadora, reversível e minimamente invasiva (Bonotto et al., 2011; Grossmann et al., 2013). A partir do momento em que tais condutas terapêuticas não fornecem os resultados esperados, com remissão significativa de sintomatologia, as abordagens terapêuticas podem evoluir para procedimentos mais invasivos, como a artroscopia (Grossmann et al., 2013).

A artroscopia é considerada um procedimento mais invasivo, quando comparada com outros procedimentos, como a artrocentese (Sakalys, Dvylys, Simuntis, \& Leketas, 2020). Esse procedimento permite uma visualização direta e manipulação das superfícies do espaço intra articulares (Sakalys et al., 2020). A artroscopia, com remoção de aderências, lavagem, manipulação e debridamento da cavidade superior da ATM, tem se mostrado uma alternativa terapêutica para deslocamentos de disco com e sem redução, não responsivos a tratamentos conservadores (Castaño-Joaqui et al., 2021; Pihut \& Gala, 2020). Injeções intra-articulares de diferentes substâncias podem ser realizadas após a artroscospia (Sakalys et al., 2020). Uma das terapias para o controle das desordens internas da ATM é conhecida como viscossuplementação, na qual é feita a infiltração de ácido hialurônico (AH) na ATM (Oliveira, Brígido, \& Saldanha, 2019; Toameh, Alkhouri, \& Karman, 2019).

$\mathrm{O}$ ácido hialurônico (AH) é um mucopolissacarídeo abundantemente encontrado no líquido sinovial, com a função de lubrificar (Kütük, Baş, Soylu, Gönen, Yilmaz, Balcioğlu, Özdamar, 2014) e nutrir os tecidos articulares. É formado por muitas unidades alternadas de ácido D-glucorônico e N-acetil-glicosamina, integrando soluções gelatinosas viscosas e hidrófilas (Bonotto et al., 2011).

O procedimento de injeção intra-articular com o sal de sódio do ácido hialurônico (AH), conhecido como hialuronato de sódio (HS) teve sua utilização primária em cavalos de corrida com desenvolvimento de artrite reumática. Em humanos, teve seu início nas grandes articulações do joelho, quadril e ombro. Somente no final da década de 70 que o HS foi empregado na ATM (Bonotto et al., 2011).

A viscossuplementação, atualmente considerada uma técnica minimamente invasiva, é realizada através da infiltração intra-articular de AH na ATM, com a finalidade precípua de eliminar ou diminuir sintomatologia dolorosa e restabelecer a 
função do aparelho mastigatório, pelo melhoramento qualitativo e quantitativo do fluido sinovial (Grossmann et al., 2013; Oliveira et al., 2019).

A técnica da viscossuplementação pode ser utilizada de forma isolada ou em conjunto com outras modalidades, sendo considerada um tratamento minimamente invasivo (Grossmann et al., 2013). Entretanto, existem ainda relatos de reações adversas ao emprego de AH combinado a técnicas minimamente invasivas ou empregado de forma isolada, ainda que descritas como leves e transitórias.

O objetivo do presente estudo foi analisar criticamente por meio de uma busca e análise da literatura nas principais bases de dados, a eficácia e segurança da realização da artroscopia associada a injeções de AH no tratamento das DTM. Sendo assim, a hipótese nula a ser refutada é de que em pacientes com diagnóstico de osteoartrite e / ou distúrbio interno da ATM refratários ao tratamento conservador, o uso de injeção intra-articular de ácido hialurônico após artroscopia na ATM não é efetivo na redução da dor e melhora dos sintomas

\section{Metodologia}

\subsection{Protocolo e registro}

O protocolo da presente revisão sistemática da literatura foi registrado junto à PROSPERO (International Prospective Register of Systematic Reviews) pelo número CRD42021258866.

Uma metodologia de busca e seleção fora empregada no preparo dessa revisão da literatura. Quatro bases de dados foram escolhidas a fim de aplicar as estratégias de busca, sendo duas internacionais MEDLINE via PUBMED, SCOPUS e duas nacionais Scielo e Bireme. A data limite de busca estipulada foi em 01 de julho de 2021.

\subsection{Estratégias de busca}

A estratégia PICO (problema, intervenção, comparação e resultado) foi definida na forma apresentada na Tabela 1.

Ao utilizar os elementos dessa estratégia foi possível elaborar a questão norteadora desse estudo, a saber: "Em pacientes com diagnóstico de osteoartrite e / ou distúrbio interno da ATM refratários ao tratamento conservador, o uso de injeção intra-articular de ácido hialurônico após artroscopia na ATM é efetivo na redução da dor e melhora dos sintomas?"

Tabela 1 - Descrição da estratégia PICO utilizada para a elaboração da questão norteadora da pesquisa.

\begin{tabular}{ll}
\hline Acrônimo & Descrição \\
\hline P (Problema) & $\begin{array}{l}\text { pacientes com diagnóstico de osteoartrite e / ou distúrbio interno da ATM refratários ao tratamento } \\
\text { conservador. }\end{array}$ \\
\hline I (Intervenção) & injeção intra-articular de AH após artroscopia na ATM \\
\hline C (Comparação) & $\begin{array}{l}\text { pacientes submetidos à artroscopia com e sem injeção de ácido hialurônico ou com injeção de outras } \\
\text { substâncias. }\end{array}$ \\
\hline O (Resultado) & redução da dor e melhora dos sintomas com injeção de ácido hialurônico após artroscopia. \\
\hline
\end{tabular}

Fonte: Autores.

Para a estratégia de busca preconizada foram utilizados termos controlados e não controlados, com o uso de operadores booleanos combinados com as palavras-chave selecionadas. Os seguintes Descritores em Ciências da Saúde DeCS/MeSH foram escolhidos: “Articulação Temporomandibular"; "Transtornos da Articulação Temporomandibular”; “Artroscopia"; "Ácido Hialurônico", bem como os respectivos termos equivalentes em inglês: "Temporomandibular Joint", “Temporomandibular Joint Disorders", “Arthroscopy”; “Hyaluronic Acid”.

As estratégias de busca nas diferentes bases de dados utilizadas constam na Tabela 2. 
Tabela 2 - Estratégias de busca nas bases de dados com termos controlados e não controlados com uso de operadores booleanos.

\begin{tabular}{|c|c|}
\hline Base de dados & Termos controlados e não controlados, com o uso de operadores booleanos \\
\hline \multirow[t]{2}{*}{ Medline via PubMed } & $\begin{array}{l}\text { ((("Temporomandibular joint disorder") AND ("Hyaluronic Acid")) AND (arthroscopy)) OR } \\
\text { (viscosupplementation) }\end{array}$ \\
\hline & $\begin{array}{l}\text { (((("Temporomandibular joint") AND ("Temporomandibular joint disorder") AND (intra- } \\
\text { articular injections)) AND ("Hyaluronic acid")) OR (viscosupplementation)) }\end{array}$ \\
\hline \multirow[t]{2}{*}{ Scopus } & $\begin{array}{l}\text { ((("Temporomandibular joint disorder") AND ("Hyaluronic Acid")) AND (arthroscopy)) OR } \\
\text { (viscosupplementation)" }\end{array}$ \\
\hline & $\begin{array}{l}\text { (((("Temporomandibular joint") AND ("Temporomandibular joint disorder") AND (intra- } \\
\text { articular injections)) AND ("Hyaluronic acid")) }\end{array}$ \\
\hline Scielo & $\begin{array}{l}\text { ((("Articulação Temporomandibular")) AND ("ácido hialurônico") AND (artroscopia)) OR } \\
\text { (viscossuplementação)" }\end{array}$ \\
\hline \multirow[t]{2}{*}{ Bireme } & $\begin{array}{l}\text { ((("Temporomandibular joint disorder") AND ("Hyaluronic Acid")) AND (arthroscopy)) OR } \\
\text { (viscosupplementation) }\end{array}$ \\
\hline & $\begin{array}{l}\text { (((("Temporomandibular joint") AND ("Temporomandibular joint disorder") AND (intra- } \\
\text { articular injections)) AND ("Hyaluronic acid")) OR (viscosupplementation)) }\end{array}$ \\
\hline
\end{tabular}

Fonte: Autores.

As próprias referencias dos artigos selecionados também foram consideradas nas buscas, com a finalidade de identificar estudos que não foram apontados nas buscas nas bases de dados.

O gerenciamento de todas as referências foi feito com um software especializado (Mendeley, New York USA) e as duplicatas foram removidas.

\subsection{Critérios de elegibilidade}

Os critérios de inclusão foram artigos redigidos em inglês, português ou espanhol, provenientes de estudos randomizados ou não, prospectivos ou retrospectivos, publicados em um intervalo de tempo de cinco anos, entre 2016 e 2021. Já os critérios de exclusão foram artigos que estudaram outra articulação do corpo humano, paciente sindrômicos ou com patologias associadas na ATM, pacientes que já foram operados na ATM, paciente com luxação redicivante, paciente não submetido a tratamento conservador prévio, pacientes menores de dezoito ou com deficiência de crescimento.

A procura por referências foi conduzida de maneira duplo cego. A seleção dos estudos foi feita em três etapas interdependentes e sucessivas. Dois pesquisadores (I.L.M. e R.G.T.) procuraram de forma independente, primeiramente, os títulos e, em seguida, os resumos de todas as referências selecionadas a partir de cada uma das bases de dados escolhidas para busca. Na etapa conseguinte, os mesmos pesquisadores avaliaram os textos completos, a fim de averiguar a adequação nos critérios de inclusão. As discordâncias ocorridas na primeira ou segunda etapa tiveram um consenso por meio de discussão e acordo mútuo entre os autores. Em situações de desacordos e/ou discrepâncias, um terceiro revisor (M.O.C.D.L.) foi chamado para que a decisão final fosse alcançada. Aquelas referências que não atenderam aos critérios de inclusão pré-estabelecidos foram excluídas em cada uma das etapas do estudo.

\subsection{Coleta dos dados}

Uma vez selecionadas referências, dois revisores (I.L.M., R.G.T.) de forma independente coletaram os dados dos estudos selecionados. Para todos os artigos incluídos, as seguintes características foram coletadas: autor, ano, tipo de estudo, metodologia de diagnóstico de DTM, tamanho amostral, AH usado, uso de outros agentes terapêuticos, tempo de acompanhamento e benefícios relatados. 


\section{Resultados}

\subsection{Seleção dos estudos}

Na primeira fase da identificação e seleção, 1298 estudos foram obtidos a partir das buscas dos termos controlados e não controlados, com operadores booleanos nas quatro bases de dados. Após a triagem inicial de títulos e resumos, restaram 34 referências. As duplicatas foram removidas e, em seguida, 22 textos completos foram lidos para avaliação de elegibilidade aos critérios de inclusão estabelecidos, sendo, posteriormente, excluídos 12 artigos. Na busca nas listas de referências dos artigos selecionados, três artigos foram identificados. Nenhuma literatura cinzenta fora selecionada. Portanto, apenas 13 artigos preencheram os critérios de elegibilidade e foram incluídos na análise qualitativa.

O fluxograma do processo de identificação, inclusão e exclusão dos estudos está demonstrado na Figura 1.

Figura 1 - Fluxograma.

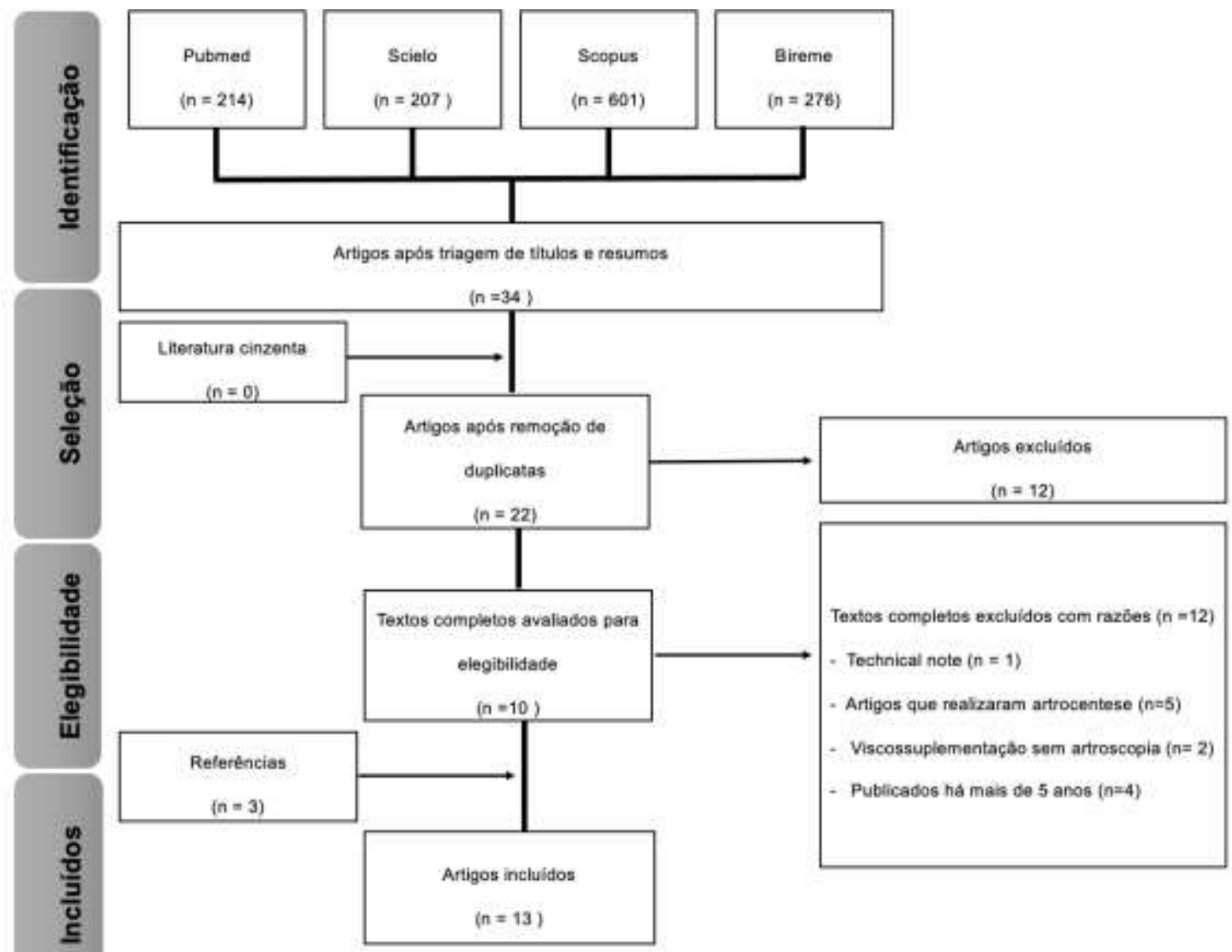

\subsection{Características dos estudos}

Fonte: Autores.

As características dos estudos incluídos estão sintetizadas na Tabela 3. 
Research, Society and Development, v. 10, n. 12, e33101219496, 2021

(CC BY 4.0) | ISSN 2525-3409 | DOI: http://dx.doi.org/10.33448/rsd-v10i12.19496

Tabela 3 - Característica avaliadas dos estudos que envolviam amostras com pacientes incluídos.

\begin{tabular}{|c|c|c|c|c|c|c|}
\hline Referência & $\begin{array}{l}\text { Metodologia de } \\
\text { diagnóstico de DTM }\end{array}$ & Tamanho da amostra & AH usado & $\begin{array}{ll}\text { Outros } & \text { agentes } \\
\text { terapêuticos } & \\
\end{array}$ & Acompanhamento & Benefícios Relatados \\
\hline $\begin{array}{l}\text { (Castaño-Joaqui et al., } \\
\text { 2021) }\end{array}$ & $\begin{array}{l}\text { Avaliação clínica em } \\
\text { estágio III e IV de } \\
\text { Wilkes }\end{array}$ & 51 & Durolane $\AA$ & Não usou & Até 12 meses & $\mathrm{NC}$ \\
\hline $\begin{array}{lr}\text { Stasko J; Statelova D, } \\
\text { Janickova } \\
\text { Mikuskova } \\
\text { Bacinsky M, Sokol J, } \\
\text { Frlickova Z, Hvizdos } \\
\text { D, 2020) }\end{array}$ & $\begin{array}{l}\text { Imagens de ressonância } \\
\text { magnética e eixo I do } \\
\text { RDC/TMD }\end{array}$ & 145 & Sinovial Mini ${ }^{\circledR}$ & Não usou & 12CAr meses & $\begin{array}{l}\text { Melhora da abertura } \\
\text { bucal máxima e escala } \\
\text { análoga de dor }\end{array}$ \\
\hline $\begin{array}{lr}\text { (Fernández-Ferro } & \mathrm{M}, \\
\text { Fernández-Sanromán } & \mathrm{J}, \\
\text { Blanco-Carrión } & \mathrm{A}, \\
\text { Costas-López } & \mathrm{A}, \\
\text { López-Betancourt } & \mathrm{A}, \\
\text { Arenaz-Bua J, 2017) } & \\
\end{array}$ & $\begin{array}{ll}\text { RDC/TDM } & \text { e imagens } \\
\text { de } & \text { ressonância } \\
\text { magnética } & \end{array}$ & 100 & Stenil@ & PRP & 18 meses & $\begin{array}{lr}\text { Injeções } & \text { intra- } \\
\text { articulares de } & \text { PRP } \\
\text { mostraram-se } & \text { mais } \\
\text { efetivas que injeções de } \\
\text { AH. }\end{array}$ \\
\hline
\end{tabular}

Fonte: Autores. 


\section{Discussão}

No presente estudo, a literatura foi revista e analisada quanto a viscossuplementação intra-articular de AH após artroscopia em pacientes com diagnóstico de DTM, refutando a hipótese nula de que em pacientes com diagnóstico de osteoartrite e / ou distúrbio interno da ATM refratários ao tratamento conservador, o uso de injeção intra-articular de ácido hialurônico após artroscopia na ATM não é efetivo na redução da dor e melhora dos sintomas. Dentre os estudos que se enquadraram nos critérios de inclusão estabelecidos, três eram estudos retrospectivos com amostras de pacientes (CastañoJoaqui et al., 2021; Fernández-Ferro, Fernández-Sanromán, Blanco-Carrión, Costas-López, López-Betancourt, Arenaz-Bua, 2017; Stasko; Statelova, Janickova, Mikuskova, Bacinsky, Sokol, Frlickova, Hvizdos, 2020), nove tratavam-se de revisões sistemáticas da literatura e /ou meta análises (Al-Moraissi, Wolford, Ellis, \& Neff, 2020; Castaño-Joaqui, Muñoz-Guerra, Campo, Martínez-Bernardini, \& Cano, 2017; Ferreira, Masterson, Lopes de Lima, de Souza Moura, Oliveira, Kelly da Silva Fidalgo, Carvalho, DosSantos, 2018; Goiato, da Silva, de Medeiros, Túrcio, \& dos Santos, 2016; Iturriaga, Bornhardt, Manterola, \& Brebi, 2017; Liu et al., 2018; Moldez, Camones, Ramos, Padilla, \& Enciso, 2018; Oliveira et al., 2019; Sakalys et al., 2020) e um estudo era de relato de caso clínico (Pastore, Goulart, Pastore, \& Prati, 2016).

O procedimento de viscossuplementação consiste na injeção intra-articular, mais especificamente a cavidade superior da ATM, com AH (Oliveira et al., 2019). O uso de AH foi associado ao aumento da produção natural de AH, inibição da progressão de osteoartrite, redução da proliferação de tecido fibroso, melhor absorção de traumas, lubrificação, efeitos antiinflamatórios, síntese de proteoglicanos e alterações na matriz cartilaginosa (Stasko; Statelova, Janickova, Mikuskova, Bacinsky, Sokol, Frlickova, Hvizdos, 2020). Ainda que tais benefícios tenham sido relatados, os estudos que preencheram os critérios de inclusão da presente revisão de literatura não avaliaram esses parâmetros histológicos. Os resultados foram mensurados por meio de dados clínicos, a saber: aumento em milímetros $(\mathrm{mm})$ da máxima abertura bucal e escala análoga visual de dor (Castaño-Joaqui et al., 2021; Fernández-Ferro, Fernández-Sanromán, Blanco-Carrión, Costas-López, LópezBetancourt, Arenaz-Bua, 2017; Stasko; Statelova, Janickova, Mikuskova, Bacinsky, Sokol, Frlickova, Hvizdos, 2020).

A maioria dos estudos analisados relata efeitos benéficos para as injeções de AH após procedimentos de artroscopia (Al-Moraissi et al., 2020; Castaño-Joaqui et al., 2017; Goiato et al., 2016; Iturriaga et al., 2017; Liu et al., 2018; Oliveira et al., 2019; Sakalys et al., 2020; Stasko; Statelova, Janickova, Mikuskova, Bacinsky, Sokol, Frlickova, Hvizdos, 2020). Os efeitos foram identificados como positivos a curto prazo (Castaño-Joaqui et al., 2017; Oliveira et al., 2019; Sakalys et al., 2020). Há ainda sugestões acerca do período em que a viscossuplementação com AH deve iniciar-se, a saber: antes da exaustão dos tratamentos conservadores ou no momento seguinte em que os pacientes mostrarem melhora da sintomatologia após tratamento inicial (Al-Moraissi et al., 2020).

Quando comparados com outras substâncias, as injeções de AH após artroscopia foram divergentes. Na comparação entre AH e corticoides, alguns autores não revelaram diferenças significantes (Goiato et al., 2016; Moldez et al., 2018), enquanto outros afirmam que AH apresentou melhores resultados (Liu et al., 2018). Ao comparar AH e PRP, o plasma rico em plaquetas teve melhor resultado, nos parâmetros escala análoga de dor e máxima abertura bucal (Fernández-Ferro M, Fernández-Sanromán, Blanco-Carrión, Costas-López, López-Betancourt, Arenaz-Bua, 2017).

Ainda que a revisão de literatura tenha sido extensa, há limitações a serem consideradas: a heterogeneidade de marcas comerciais e posologia (número de doses e injeções) de AH. Nos estudos de ensaio clínico foram identificadas três marcas comerciais distintas (Durolane ${ }^{\circledR}$, Sinovial Mini® e Stenil®) (Castaño-Joaqui et al., 2021; Fernández-Ferro, FernándezSanromán, Blanco-Carrión, Costas-López, López-Betancourt, Arenaz-Bua, 2017; Stasko; Statelova, Janickova, Mikuskova, Bacinsky, Sokol, Frlickova, Hvizdos, 2020), com protocolos variados de injeção intra-articular nos pacientes. O acompanhamento dos pacientes foi de no máximo 18 meses, o que dificulta a interpretação dos resultados a longo prazo. Além disso, esta revisão da literatura incluiu um número limitado de estudos relevantes com tamanhos de amostra relativamente 
pequenos, uma vez que preenchiam os critérios de inclusão.

Poucos estudos que referenciavam o procedimento de artroscopia associado à injeção de AH isoladamente (CastañoJoaqui et al., 2021). A maioria dos estudos tratava a artroscopia como mais uma modalidade de procedimento cirúrgico para a ATM a ser comparada, resultando em um viés para a presente revisão da literatura.

Diante do exposto, emerge a necessidade de se realizar um procedimento operacional padrão para as injeções intraarticulares de AH para pacientes com DTM, abrangendo o diagnóstico de DTM, o período de acompanhamento do paciente, os exames de imagem a serem solicitados antes e após o procedimento, bem como os critérios clínicos a serem avaliados.

\section{Conclusão}

As evidências coletadas e analisadas nesse estudo permitiram concluir que:

- A viscossuplementação de AH após artroscopia mostrou-se com mais evidências científicas de benefícios clínicos que contraindicações ou efeitos adversos;

- As injeções de AH concomitantes ao procedimento de artroscopia demonstraram ser efetivas a curto prazo para melhora de quadros clínicos de dor e máxima abertura bucal;

- Faz-se necessário o estabelecimento de um protocolo e procedimento operacional padrão para o uso de AH, quanto ao tipo, concentração, dose e posologia.

\section{Referências}

Al-Moraissi, E. A., Wolford, L. M., Ellis, E., \& Neff, A. (2020). The hierarchy of different treatments for arthrogenous temporomandibular disorders: A network meta-analysis of randomized clinical trials. Journal of Cranio-Maxillofacial Surgery, 48(1), 9-23. https://doi.org/10.1016/j.jcms.2019.10.004

Bonotto, D., Custódio, L. G., \& Cunali, P. A. (2011). Viscossuplementação como tratamento das alterações internas da articulação temporomandibular: relato de casos. Revista Dor, 12(3), 274-278. https://doi.org/10.1590/s1806-00132011000300016

Castaño-Joaqui, O. G., Cano-Sánchez, J., Campo-Trapero, J., \& Muñoz-Guerra, M. F. (2021). TMJ arthroscopy with hyaluronic acid: A 12-month randomized clinical trial. Oral Diseases, 27(2), 301-311. https://doi.org/10.1111/odi.13524

Castaño-Joaqui, O. G., Muñoz-Guerra, M. F., Campo, J., Martínez-Bernardini, G., \& Cano, J. (2017). Estado actual de la viscosuplementación con ácido hialurónico en el tratamiento de los trastornos temporomandibulares: revisión sistemática. Revista Espanola de Cirugia Oral y Maxilofacial, 39(4), 213-220. https://doi.org/10.1016/j.maxilo.2016.11.002

Cömert Kiliç S, Güngörmüş M, S. M. (2015). Is arthrocentesis plus platelet- rich plasma superior to arthrocentesis plus hyaluronic acid for the treatment of temporomandibular joint osteoarthritis : a randomized clinical trial. Int. J. Oral Maxillofac. Surg, 73(8), 1473-1483. https://doi.org/10.1016/j.ijom.2016.06.009

Fernández-Ferro M, Fernández-Sanromán J, Blanco-Carrión A, Costas-López A, López-Betancourt A, Arenaz-Bua J, S. M. B. (2017). Comparison of intraarticular injection of plasma rich in growth factors versus hyaluronic acid following arthroscopy in the treatment of temporomandibular dysfunction: A randomised prospective study. J Craniomaxillofac Surg, 45(4), 449-454. https://doi.org/10.1016/j.jcms.2017.01.010

Ferreira N, Masterson D, Lopes de Lima R, de Souza Moura B, Oliveira AT, Kelly da Silva Fidalgo T, Carvalho ACP, DosSantos MF, G. E. (2018). Efficacy of viscosupplementation with hyaluronic acid in temporomandibular disorders: A systematic review. Journal of Cranio-Maxillo-Facial Surgery, 46(11), 19431952. https://doi.org/10.1016/j.jcms.2018.08.007.This

Goiato, M. C., da Silva, E. V. F., de Medeiros, R. A., Túrcio, K. H. L., \& dos Santos, D. M. (2016). Are intra-articular injections of hyaluronic acid effective for the treatment of temporomandibular disorders? A systematic review. International Journal of Oral and Maxillofacial Surgery, $45(12), 1531-1537$. https://doi.org/10.1016/j.ijom.2016.06.004

Grossmann, E., Januzzi, E., \& Iwaki Filho, L. (2013). O uso do hialuronato de sódio no tratamento das disfunções temporomandibulares articulares. Revista Dor, 14(4), 301-306. https://doi.org/10.1590/s1806-00132013000400013

Iturriaga, V., Bornhardt, T., Manterola, C., \& Brebi, P. (2017). Effect of hyaluronic acid on the regulation of inflammatory mediators in osteoarthritis of the temporomandibular joint: a systematic review. International Journal of Oral and Maxillofacial Surgery, $46(5)$, 590-595. https://doi.org/10.1016/j.ijom.2017.01.014

Kütük N, Baş B, Soylu E, Gönen Z. B, Yilmaz C, Balcioğlu E, Özdamar S, A. A. (2014). Effect of Platelet-Rich Plasma on Fibrocartilage , Cartilage , and Bone Repair in Temporomandibular Joint. J Oral Maxillofac Surg., 72(2), 277-284. https://doi.org/10.1016/j.joms.2013.09.011

Liu, Y., Wu, J., Fei, W., Cen, X., Xiong, Y., Wang, S., \& Liang, X. (2018). Is There a Difference in Intra-Articular Injections of Corticosteroids, Hyaluronate, or Placebo for Temporomandibular Osteoarthritis? In Journal of Oral and Maxillofacial Surgery (Vol. 76). https://doi.org/10.1016/j.joms.2017.10.028 
Research, Society and Development, v. 10, n. 12, e33101219496, 2021

(CC BY 4.0) | ISSN 2525-3409 | DOI: http://dx.doi.org/10.33448/rsd-v10i12.19496

Moldez, M., Camones, V., Ramos, G., Padilla, M., \& Enciso, R. (2018). Effectiveness of Intra-Articular Injections of Sodium Hyaluronate or Corticosteroids for Intracapsular Temporomandibular Disorders: A Systematic Review and Meta-Analysis. Journal of Oral \& Facial Pain and Headache, 32(1), 53-66. https://doi.org/10.11607/ofph.1783

Oliveira, L. E. A. de, Brígido, J. A., \& Saldanha, A. D. D. (2019). Effects of the hyaluronic acid infiltration in the treatment of internal temporomandibular joint disorders. Brazilian Journal Of Pain, 2(2), 182-186. https://doi.org/10.5935/2595-0118.20190032

Pastore, G. P., Goulart, D. R., Pastore, P. R., \& Prati, A. J. (2016). Removal of a solitary synovial chondromatosis of the temporomandibular joint using arthroscopy. Journal of Craniofacial Surgery, 27(4), 967-969. https://doi.org/10.1097/SCS.0000000000002612

Pihut, M., \& Gala, A. (2020). The Application of Intra-Articulr Injections for Management of the Consequences of Disc Displacement without Reduction. Int. J. Environ. Res. Public Health, 17(4726), 1-10.

Sakalys, D., Dvylys, D., Simuntis, R., \& Leketas, M. (2020). Comparison of Different Intraarticular Injection Substances Followed by Temporomandibular Joint Arthroscopy. The Journal of Craniofacial Surgery, 31(3), 637-641. https://doi.org/10.1097/SCS.0000000000006098

Sousa, B. M., López-Valverde, N., López-Valverde, A., Caramelo, F., Fraile, J. F., Payo, J. H. R. M. (2020). Different Treatments in Patients with Temporomandibular Joint Disorders : A Comparative Randomized Study. Medicina (Kaunas), 56(3), 113. https://doi.org/10.3390/medicina56030113

Stasko J; Statelova D, Janickova M, Mikuskova K, Bacinsky M, Sokol J, Frlickova Z, Hvizdos D, M. I. (2020). Hyaluronic acid application vs arthroscopy in treatment of internal temporomandibular joint disorders. Bratisl Med J, 121(5), 352-357. https://doi.org/10.4149/BLL

Toameh, M. H., Alkhouri, I., \& Karman, M. A. (2019). Management of patients with disk displacement without reduction of the temporomandibular joint by arthrocentesis alone, plus hyaluronic acid or plus platelet-rich plasma. Dent Med Probl, 56(3), 265-272. https://doi.org/10.17219/dmp/109329 